\title{
MiR-138 is a potent regulator of the heterogenous MYC transcript population in cancers
}

\author{
Ng Desi $\mathbb{D}^{1,2}$, Velda Teh ${ }^{1}$, Qing Yun Tong ${ }^{1}$, Chun You Lim ${ }^{1}$, Hossein Tabatabaeian ${ }^{1}$, Xiao Hong Chew ${ }^{1}$, Avencia Sanchez-Mejias ${ }^{1,6}$, \\ Jia Jia Chan (iD ${ }^{1}$, Bin Zhang ${ }^{1}$, Priyankaa Pitcheshwar ${ }^{1}$, Bei-En Siew ${ }^{3}$, Shi Wang ${ }^{4}$, Kuok-Chung Lee ${ }^{5}$, Choon-Seng Chong (iD ${ }^{3,5}$, \\ Wai-Kit Cheong ${ }^{5}$, Bettina Lieske, ${ }^{3,5}$, lan Jse-Wei $\operatorname{Tan}^{5}$, Ker-Kan $\operatorname{Tan}^{3,5}$ and Yvonne Tay (D) ${ }^{1,2}$
}

(c) The Author(s) 2021

\begin{abstract}
$3^{\prime}$ UTR shortening in cancer has been shown to activate oncogenes, partly through the loss of microRNA-mediated repression. This suggests that many reported microRNA-oncogene target interactions may not be present in cancer cells. One of the most wellstudied oncogenes is the transcription factor MYC, which is overexpressed in more than half of all cancers. MYC overexpression is not always accompanied by underlying genetic aberrations. In this study, we demonstrate that the MYC $3^{\prime} U T R$ is shortened in colorectal cancer (CRC). Using unbiased computational and experimental approaches, we identify and validate microRNAs that target the MYC coding region. In particular, we show that miR-138 inhibits MYC expression and suppresses tumor growth of CRC and hepatocellular carcinoma (HCC) cell lines. Critically, the intravenous administration of miR-138 significantly impedes MYCdriven tumor growth in vivo. Taken together, our results highlight the previously uncharacterized shortening of the MYC $3^{\prime} U T R$ in cancer, and identify miR-138 as a potent regulator of the heterogenous MYC transcript population.
\end{abstract}

Oncogene (2022) 41:1178-1189; https://doi.org/10.1038/s41388-021-02084-x

\section{INTRODUCTION}

More than half of protein-coding genes produce messenger RNA (mRNA) transcripts with alternative $3^{\prime}$ untranslated regions (UTRs), which contain binding sites for post-transcriptional regulators such as microRNAs (miRNAs). Alternative $3^{\prime}$ UTRs thus comprise varying landscapes of miRNA response elements (MREs), enabling them to differentially regulate the stability and translational efficiency of their cognate mRNAs. In cancer cells, widespread shortening of 3'UTRs by alternative polyadenylation (APA) has been shown to contribute to tumorigenesis by controlling the expression of oncogenes in the absence of genetic alterations, in part by enabling them to escape miRNA-mediated regulation [1-4]. Critically, the vast majority of miRNA studies have focused on MREs located in the 3'UTRs of target mRNAs. The observation that $3^{\prime} U T R$ shortening is increased in cancers suggests that many known miRNA:target interactions may be lost in cancer cells [3, 4], and highlights the need to better understand how established cancer driver genes with shortened $3^{\prime}$ UTRs may be regulated in cancer cells.

In this study, we focus on the transcription factor MYC, which is overexpressed in more than half of all cancers $[5,6]$. Intriguingly, although MYC is overexpressed 5 to 400 -fold in $70 \%$ of colorectal carcinoma (CRC) patients and 4 to 6 -fold in $44 \%$ of hepatocellular carcinoma (HCC) patients, genetic amplification of MYC is only detected in $10 \%$ and $4 \%$ of CRC and HCC patients respectively [7-11]. These observations that MYC overexpression is not always accompanied by underlying genetic aberrations suggest that post- transcriptional mechanisms may play key roles in regulating MYC expression and function in these cancers.

Previously, a MYC construct containing its 3'UTR was shown to have lower MYC protein expression compared to those without, demonstrating the importance of the $3^{\prime} U T R$ in regulating MYC expression [12]. Multiple studies have identified miRNAs that bind to the MYC 3'UTR and regulate MYC expression [13-15]. In contrast, only a few miRNAs that target the MYC coding sequence (CDS) have been described. Specifically, miR-184 and miR-320b, as well as miR-320a and miR-744, were shown to inhibit MYC expression in CRC $[16,17]$ and HCC respectively $[18,19]$. However, their potential efficacy as treatment options in MYC-driven cancers remains to be explored.

In this study, we find that the MYC $3^{\prime}$ UTR is shortened in cancer, and integrate two complementary computational and biochemical approaches to identify miRNAs that target the MYC coding region as this is present in all MYC transcript variants. In particular, we show that miR-138 binds to the MYC CDS, inhibits MYC expression and suppresses tumor growth. Critically, the intravenous administration of miR-138 significantly impedes the tumor growth of MYC-driven mouse model. These results highlight the previously uncharacterized shortening of the MYC $3^{\prime}$ UTR in cancer, and identify miR-138 as a potent regulator of the heterogenous MYC transcript population. These data may open new avenues for the treatment of MYC-driven cancers.

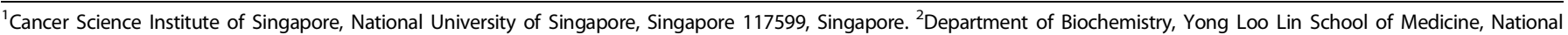

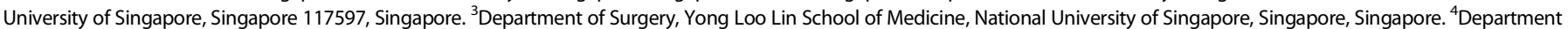

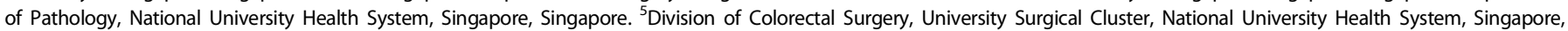
Singapore. ${ }^{6}$ Present address: Department of Experimental and Health Sciences, Pompeu Fabra University, 08003 Barcelona, Spain. ${ }^{\circledR e m a i l: ~ y v o n n e t a y @ n u s . e d u . s g ~}$ 
A

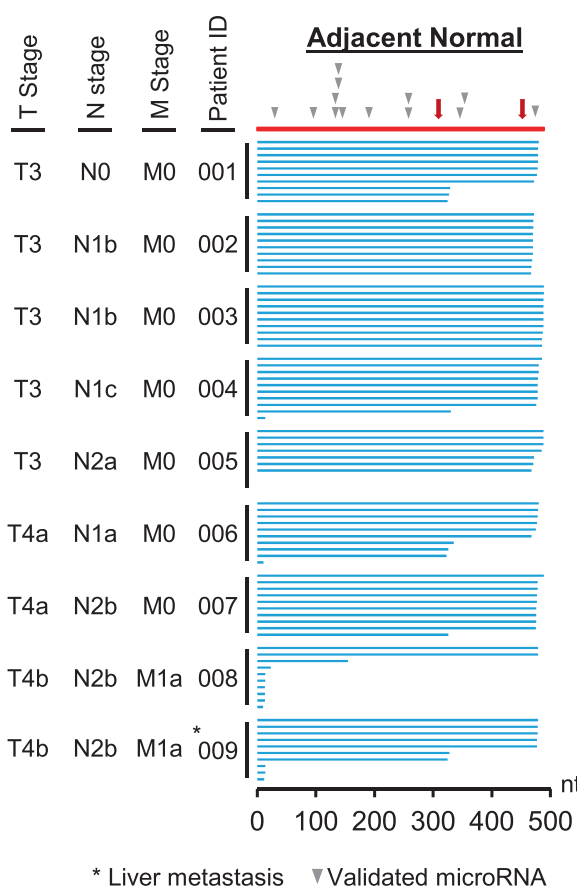

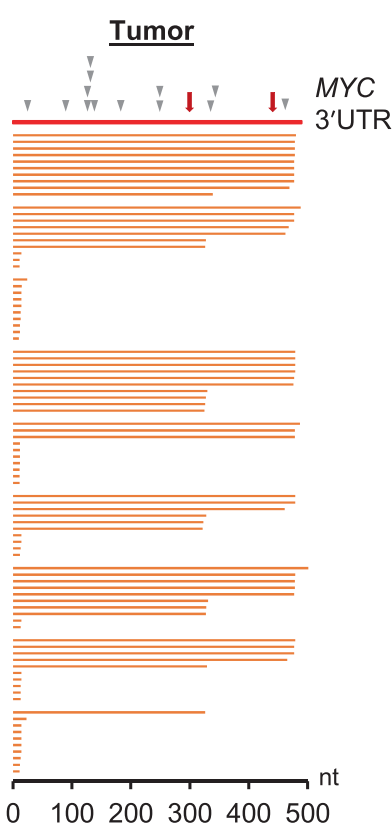

B

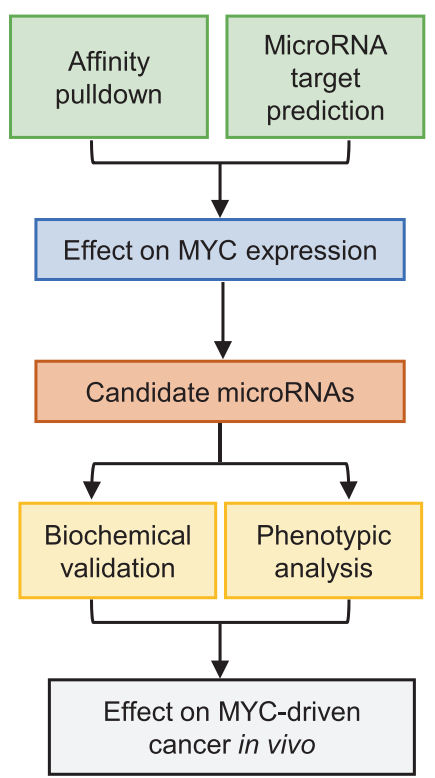

C

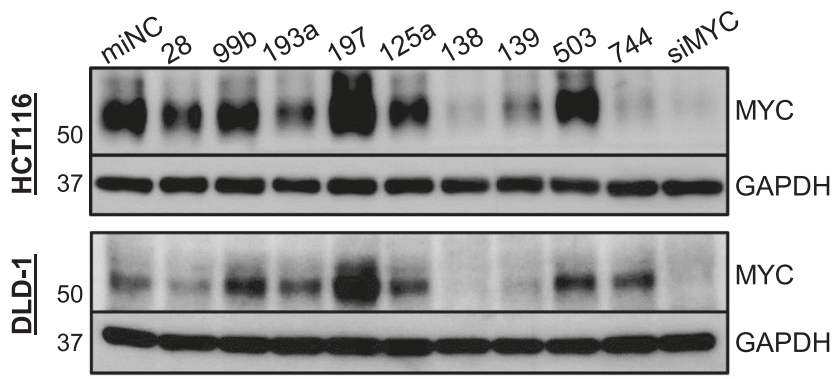

-HCT116 aDLD-1

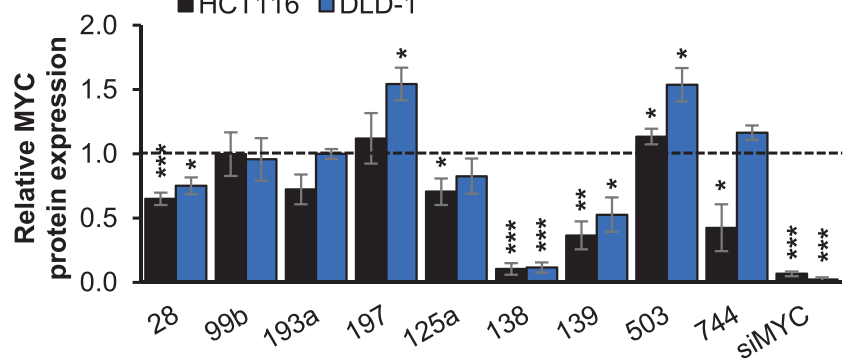

D

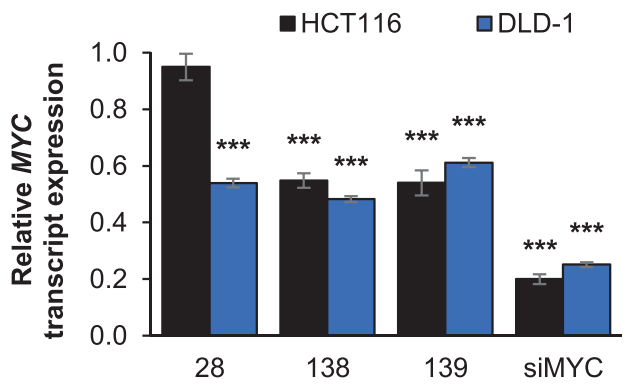

E

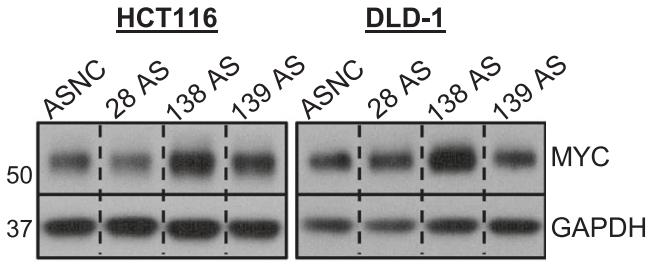

$\mathbf{F}$

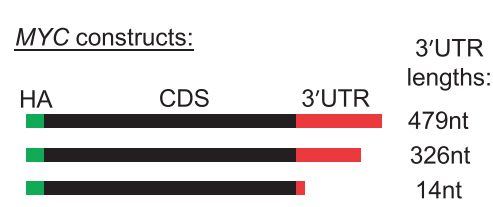

G

HCT116

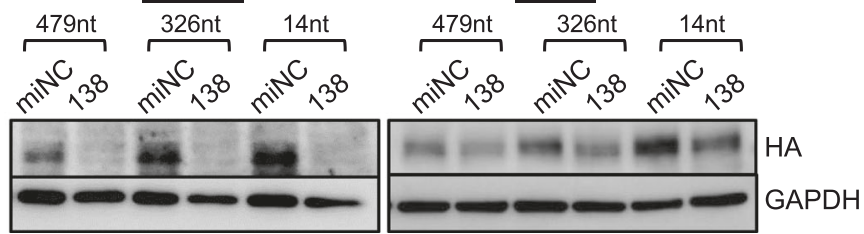

\section{RESULTS}

Identification of potential MYC CDS-targeting miRNAs

The overwhelming majority of identified miRNA:MYC interactions are located in its $3^{\prime}$ UTR. However, reports describing widespread $3^{\prime}$ UTR shortening in cancers suggest that these interactions may not exist in cancer cells, contributing to the overexpression of MYC in multiple cancers often in the absence of genetic aberrations. To investigate this, we first examined potential APA of the MYC $3^{\prime} U T R$ in tumor and adjacent normal samples from CRC patients (primary tumor stage (T stage) $>2$ ) using $3^{\prime}$ Rapid Amplification of cDNA 
Fig. 1 Identification of potential MYC CDS-targeting miRNAs. A Schematic representation of MYC 3'UTR isoforms detected by $3^{\prime}$ RACE in nine pairs of CRC tumor and adjacent normal patient samples $(N=9)$. B Flowchart outlining the workflow to identify and functionally characterize miRNAs that target the MYC CDS. MiRNAs were identified via affinity pulldown by MS2-tagged RNA Affinity Purification (MS2TRAP) and miRNA target prediction by RNA22. C Western blot (top panel) and densitometry quantification (bottom panel) of MYC protein expression upon the overexpression of candidate miRNAs in HCT116 and DLD-1 cell lines. D RT-qPCR analysis of MYC transcript expression upon the overexpression of miR-28-5p, miR-138 and miR-139-3p in HCT116 and DLD-1 cell lines. E Western blot analysis of MYC protein expression upon miRNA inhibition by antisense miRNA inhibitors (AS) in HCT116 (left panel) and DLD-1 (right panel). F Schematic illustrating the HA-tagged MYC constructs with different $3^{\prime}$ UTR lengths. G Western blot analysis of HA-tagged MYC protein expression upon miR-138 overexpression (138) in HCT116 (left panel) and DLD-1 (right panel). Mean \pm SEM; $N \geq 3$. ${ }^{*} P<0.05 ;{ }^{* *} P<0.01 ;{ }^{* * *} P<0.001$.

Ends ( $3^{\prime}$ RACE) $(N=9)$. Three main MYC $3^{\prime} U T R$ isoforms were detected, two corresponding to annotated poly $(A)$ signals and one in close proximity to the stop codon (Fig. 1A). Interestingly, more MYC $3^{\prime}$ UTR shortening was observed in the tumor samples than in the adjacent normal samples, and in patients with cancer at the regional lymph nodes ( $\mathrm{N}$ stage $>0$ ). We postulate that MYC $3^{\prime} \mathrm{UTR}$ shortening will result in a loss of miRNA binding. In addition, this data suggests that studying the post-transcriptional regulation of the MYC CDS may lead to the identification of crucial miRNAs that modulate the expression of all MYC transcript variants, including those which are predominantly expressed in advanced cancer.

Two approaches were employed to systematically identify miRNAs that target the MYC CDS: affinity pulldown by MS2-tagged RNA affinity purification (MS2-TRAP) coupled with highthroughput $\mathrm{qPCR}$ and the miRNA target prediction by RNA22 (Fig. 1B) [20]. MiR-193a-5p, miR-197-3p, miR-28-5p and miR-99b$3 p$ were shortlisted from the high-throughput $q P C R$ analysis based on their consistent enrichment (>1.5) (Supplementary Fig. S1a and Supplementary Data File S1). The second shortlisting criterion was the downregulation of their expression in CRC (Supplementary Fig. S1b) as the MS2-TRAP was performed using the CRC cell line HCT116. Although the MS2-TRAP-qPCR approach allows for the identification of miRNAs which associate with the MYC CDS in a specific cell-type, it may not enrich for miRNAs that are lowly expressed in the particular cell line studied. As a complementary approach, we performed miRNA target prediction using RNA22. MiR-125a-3p, miR-138-5p (commonly known as miR-138), miR139-3p, miR-503-5p and miR-744-5p (commonly known as miR744) were shortlisted based on their predicted binding to conserved sites on both the human and mouse MYC CDS (Supplementary Table S1), as well as their downregulation in CRC (Supplementary Fig. S1c) and HCC (Supplementary Fig. S1d). As mentioned, miR-744 has been validated to inhibit MYC expression in HCC [19], therefore, it was included in this study as a positive control and to explore its regulation of MYC in CRC.

To study the effect of the shortlisted miRNAs on MYC expression, we overexpressed the individual miRNAs in HCT116 and DLD-1 which are commonly used CRC cell lines. In addition, both HCT116 and DLD-1 have elevated expression of MYC relative to two normal colon cell lines, CCD-18Co and CCD841CoN (Supplementary Fig. S1e). We found that only the overexpression of miR-28-5p (28), miR-138 (138) and miR-139-3p (139) consistently and significantly decreased MYC protein and transcript expression in both HCT116 and DLD-1 (Fig. 1C and D). Interestingly, these effects were not observed for miR-744, suggesting that its regulatory effect on MYC is cell-type specific. As a reciprocal approach, we next determined the effect of miR28-5p, miR-138 and miR-139-3p inhibition on MYC expression using antisense miRNA inhibitors (AS). The inhibition of miR-138 significantly increased MYC protein expression, whereas miR-28$5 p$ and miR-139-3p inhibition had no effect on MYC (Fig. 1E and Supplementary Fig. S1f). Based on these observations, we focused on miR-138 for subsequent experimental validation.

To investigate whether miR-138 could regulate MYC transcripts with varying $3^{\prime} U T R$ lengths, we cloned the MYC $3^{\prime} U T R$ isoforms downstream of the MYC CDS with a HA tag at the N-terminus
(Fig. 1F). We found that miR-138 overexpression downregulated the expression of all HA-tagged MYC isoforms (Fig. 1G), demonstrating its ability to inhibit the expression of heterogenous MYC transcript isoforms.

\section{Validation of miR-138 as a MYC CDS-targeting miRNA}

MiR-138 is predicted to have three MREs on the MYC CDS, denoted as MRE A, MRE B and MRE C (Fig. 2A and Supplementary Table S2). Both human and mouse miR-138 MREs are predicted to be at the same positions with similar nucleotide sequences, suggesting that miR-138-mediated regulation of the MYC CDS may be conserved in these two species (Supplementary Table S2). To validate these predicted MREs, we first performed MRE-luciferase reporter assays. The overexpression of miR-138 significantly and consistently decreased the relative luciferase activities of MRE A and MRE C, but did not have a consistent effect on MRE B in both HCT116 and DLD-1 (Fig. 2B). To further validate the regulation of the MYC CDS, we cloned the ATG-less MYC CDS downstream of the Renilla luciferase $(R L U C)$ gene, after the stop codon. We also mutated the sequence corresponding to the miR-138 seed region within each MRE on a MYC CDS-luciferase reporter construct (Fig. 2C). We found that mutant MRE A (CDS mut_A), but not mutant MRE $C$ (CDS_mut C), abolished miR-138-mediated repression of the MYC CDS (Fig. 2D). This suggests that MRE A, but not MRE C, is critical for miR-138 regulation of MYC. In support of this, we found that MRE A was also identified by two other miRNA target prediction platforms, STarMir (Supplementary Table S3) and miRDB (Supplementary Data File S2).

To recapitulate the regulatory effect of miR-138 on the CDS of its target transcript, we inserted the miR-138 MRE A at the $3^{\prime}$ end of the RLuc gene, in-frame before the stop codon (Fig. 2E). Consistently, the overexpression of miR-138 significantly downregulated the relative luciferase activity of MRE $A$. In addition, we created a fusion RLuc-MYC gene by cloning the wild-type (WT) MYC CDS or MYC CDS with mutated MRE A (mut_A) downstream of and in-frame with the RLuc gene (Fig. 2F). We found that the mutation of miR-138 MRE A on the MYC CDS diminished miR-138mediated regulation of the MYC CDS. Next, we performed biotinylated miR-138 pulldowns and observed a significant enrichment of the MYC transcript (Fig. 2G). Taken together, these data provide support for the regulation of the MYC CDS by miR138 via MRE A.

\section{MiR-138 regulates MYC target gene expression}

Next, we explored the effect of miR-138 on MYC target gene expression. MYC has been shown to induce the expression of genes which are involved in cell cycle progression, such as CDK4 and CDK6 [21, 22], as well as to repress those involved in cell cycle arrest such as p27 [23]. Repression of CDK4 and CDK6 levels by miR-138 has been demonstrated in breast cancer cell lines [24]. However, direct regulation was only validated for CDK6 in glioblastoma multiforme [25]. We performed western blot analysis to investigate if these interactions were present in CRC, and observed a significant reduction in CDK4 and CDK6 protein levels upon miR-138 overexpression (Fig. 3A). In addition, we found that the CDK6 transcript was significantly enriched in the biotinylated 
A

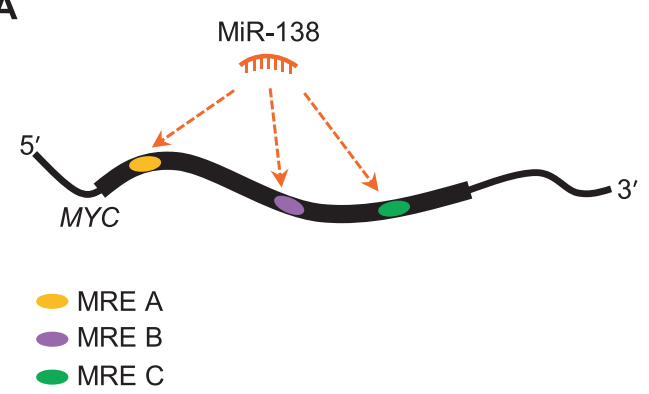

B

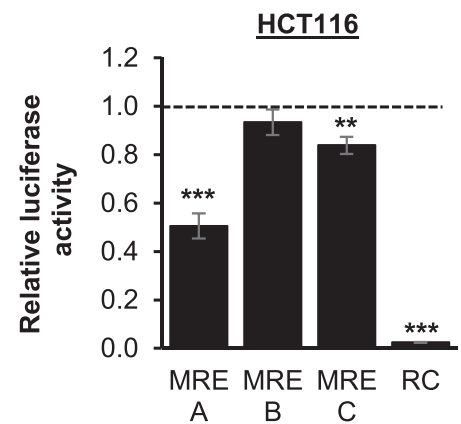

$\underline{\text { DLD-1 }}$

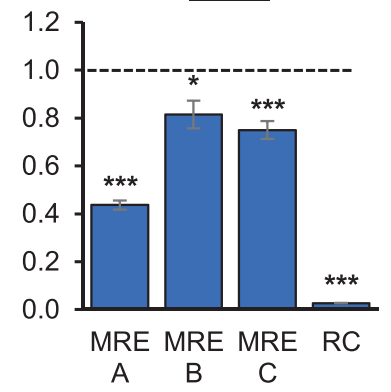

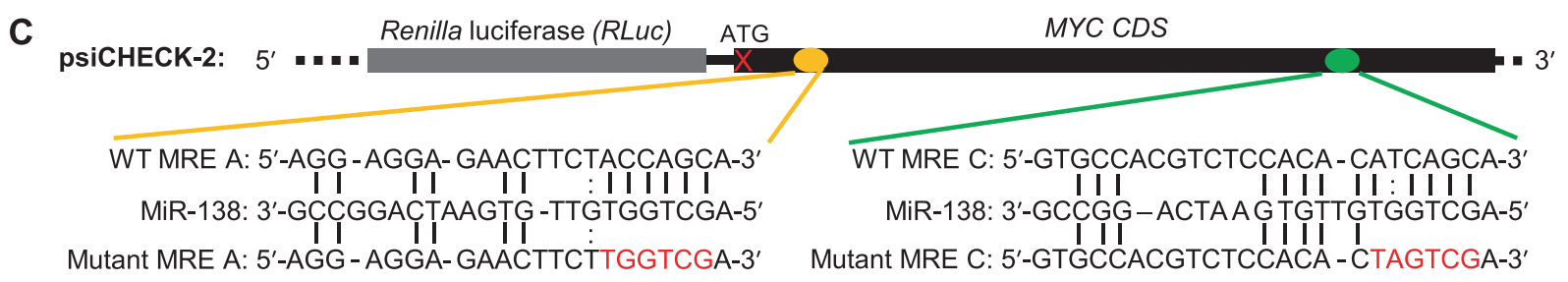

D

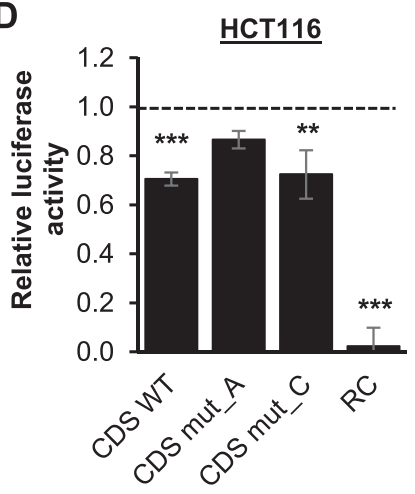

DLD-1

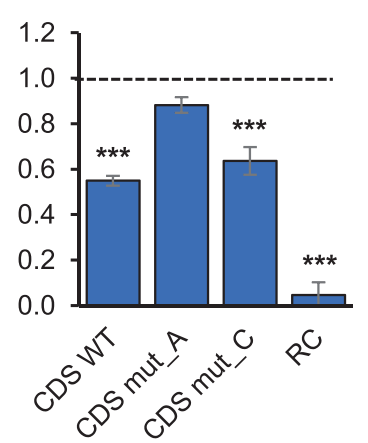

$\mathbf{F}$

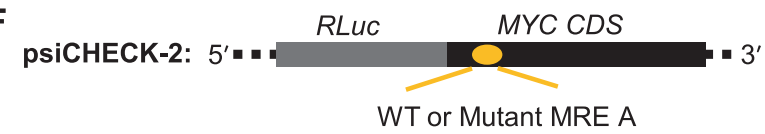

$E_{p s i c}$

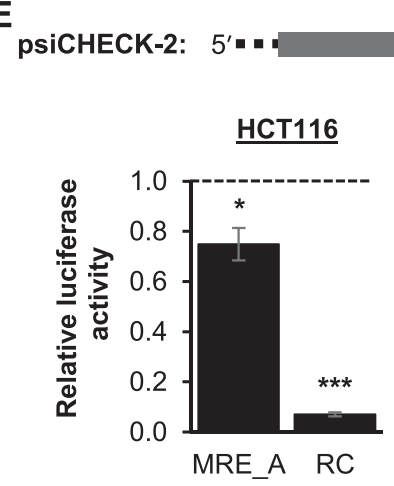

G

\section{RLUC}

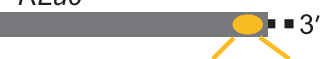

MRE A

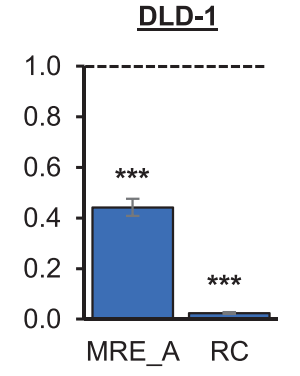

DLD-1
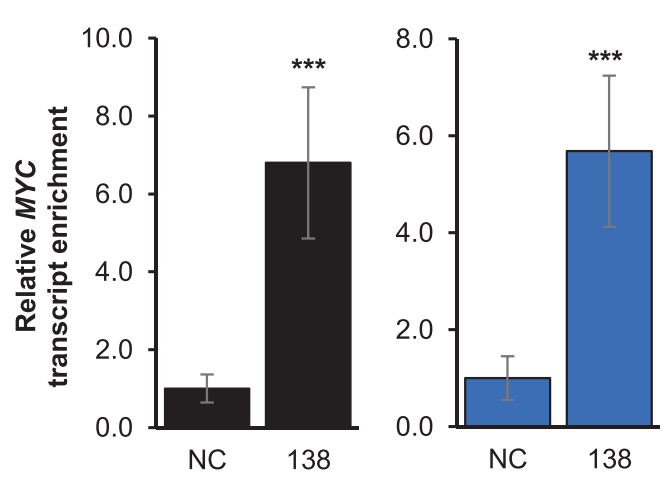

miR-138 pulldown in both CRC cell lines, while the CDK4 transcript was only enriched in HCT116 (Fig. 3B). This suggests that the regulation of CDK4 by miR-138 might be indirect and/or cell-line specific. Next, we performed MRE-luciferase reporter assays using the validated WT miR-138 MRE on the CDK6 transcript and a version with mutations in the miR-138 seed region (Fig. 3C). Consistent with other findings, the overexpression of miR-138 decreased the relative luciferase activity of the WT MRE, while this effect was abolished in the mutant (Fig. 3C) [25]. Overall, our data confirmed that CDK6 was also a direct target of miR-138 in CRC.

Based on in-silico target identification, we identified and validated LYPLA1 as another direct target of miR-138 in CRC (Supplementary Fig. S2a-f). We also confirmed the growthpromoting role of LYPLA1 in CRC (Supplementary Fig. S2g), which was consistent with its previously described role in non-small cell lung cancer (NSCLC) progression [26]. 
Fig. 2 Validation of miR-138 as a MYC CDS-targeting miRNA. A Schematic representation of predicted miR-138 MREs on the MYC transcript. The thick line indicates the MYC CDS, while the thin lines indicate the UTRs. B Effect of miR-138 overexpression on the luciferase activity of the respective MRE-reporter constructs in HCT116 (left panel) and DLD-1 (right panel) cell lines. RC denotes the reverse complement of miR-138 which was used as positive control. C Schematic representation of the ATG-less MYC CDS cloned into the psiCHECK-2 vector. Location and sequences of the wild-type (WT) and mutant MREs A (yellow oval) and C (green oval) are depicted. Red font indicates the mutated nucleotides. The cross indicates the absence of the start codon ATG. D Effect of miR-138 overexpression on the luciferase activity of the MYC CDS reporter constructs with mutated MREs (CDS mut) compared to the wild-type CDS reporter (CDS WT) in HCT116 (left panel) and DLD-1 (right panel) cell lines. E Effect of miR-138 overexpression on the luciferase activity of the Renilla luciferase (RLuc) gene fused with MRE A in HCT116 (left panel) and DLD-1 (right panel) cell lines. Top panel illustrates the modification of RLuc gene construct. F Effect of miR-138 overexpression on the luciferase activity of the RLUC and MYC CDS fusion reporter construct with mutated MRE A (mut_A) compared to the wild-type MYC CDS (WT) in the HCT116 (left panel) and DLD-1 (right panel) cell lines. The top panel illustrates the fusion gene construct. G RTqPCR analysis of MYC transcript enrichment upon biotinylated miR-138 pulldown in HCT116 (left panel) and DLD-1 (right panel) cell lines. (B, D, E and F) Mean \pm SEM; $N \geq 3$. G Mean \pm STD; $N \geq 3$. ${ }^{*} P<0.05 ; * * P<0.01 ;{ }^{* * *} P<0.001$.

In addition, miR-138 overexpression increased p27 protein expression (Fig. 3A), but its transcript was not enriched in the biotinylated miR-138 pulldown (Fig. 3B). Further examination showed that the expression of p27 was significantly upregulated upon MYC knockdown in both HCT116 and DLD-1 cells, suggesting that miR-138 exerts an indirect effect on p27 expression via MYC (Fig. 3D). Taken together, miR-138 may have a strong tumor-suppressive effect in CRC by targeting several transcripts involved in cell cycle progression either directly or indirectly via MYC.

\section{MiR-138 possesses tumor-suppressive properties in CRC}

The decreased expression of miR-138 in CRC tissues compared to normal colon has been described previously and was found to be associated with a lower degree of malignancy and better survival of CRC patients [27]. Consistent with this data, we found that miR138 was downregulated in the CRC tumors of our cohort compared to the adjacent normal tissue (Fig. 4A). Critically, there was a significant negative correlation between miR-138 and MYC transcript expression in these patients (Fig. 4B). Taken together with our earlier data (Fig. 1C-E), these findings suggest that the downregulation of miR-138 might contribute to the elevated MYC expression in CRC patients.

Upregulation of miR-138 significantly decreased the cell viability of both CRC cell lines (Fig. 4C). In addition, the overexpression of miR-138 decreased (Fig. 4D and E), while its inhibition increased (Fig. 4F and $\mathrm{G}$ ) both anchorage-dependent and -independent growth of CRC cells. These observations are consistent with several previous reports, and collectively suggest that miR-138 has potent tumor-suppressive properties in CRC [28-30].

\section{MiR-138 consistently inhibits MYC expression and cell proliferation in other cancers}

MicroRNA regulation can be highly context-specific, and miR-138 has been shown to exhibit oncogenic properties in other cancers such as malignant glioma [31] and triple-negative breast cancer [32]. Thus, we next explored whether miR-138-mediated regulation of MYC extended to other cancers. Based on TCGA expression data, we selected liver HCC, kidney clear cell renal cell carcinoma and lung squamous cell carcinoma for further study as miR-138 was significantly downregulated in these cancers (Fig. $5 \mathrm{~A}$ and Supplementary Fig. S3a) [33]. In accordance with our data in CRC cell lines, overexpression of miR-138 consistently downregulated MYC expression (Fig. 5B and Supplementary Fig. S3b) and in vitro cell growth (Fig. 5C, D and Supplementary Fig. S3C and S3d) of all three cancer types.

\section{MiR-138 suppresses MYC-driven carcinogenesis in vivo}

The potent repressive effect of miR-138 on MYC expression in cancer cell lines led us to hypothesize that it may also have a significant effect on MYC-driven carcinogenesis in vivo. Of the four cancer types studied in vitro, we focused on liver cancer, which has frequent overexpression of MYC (Supplementary Fig. S3e) without genetic amplification [9, 10]. In addition, MYC is a wellestablished driver of liver cancer initiation and progression, so the identification of novel MYC-regulatory pathways in this cancer may have profound scientific and clinical impact [34, 35]. Further in vitro analyses also showed that MYC expression (Fig. 5E and Supplementary Fig. S3f) and cell growth (Fig. 5F, G and Supplementary Fig. S3g) were upregulated upon miR-138 inhibition in HCC cell lines.

To investigate the growth inhibitory effect of miR-138 in vivo, we performed weekly intravenous miR-138 injections in LAP-tTA X tet-o-MYC transgenic mice which overexpresses human MYC CDS in the liver and is widely used to study MYC-driven HCC (Fig. 6A) [36]. Mice were sacrificed a week after the last injection. The mice injected with miR-138 mimics had significantly elevated miR-138 levels in their livers (Fig. 6B) and other organs (Supplementary Fig. S4a), and decreased Myc transcript expression compared to those injected with the negative control (Supplementary Fig. S4b). Strikingly, the overexpression of miR-138 in the LAP-tTA X tet-oMYC mice effectively reduced both MYC transcript and protein levels (Fig. 6C, D and Supplementary Fig. S4C), as well as liver tumor growth compared to the negative control (miNC) (Fig. 6E). Collectively, our results highlight the potential utility of miR-138 as a previously uncharacterized inhibitor of MYC-driven carcinogenesis in vivo.

\section{DISCUSSION}

Although widespread $3^{\prime}$ UTR shortening has been shown to result in the loss of miRNA targeting, and associated disruption of competing endogenous RNA networks, little is known about the specific $3^{\prime}$ UTR populations of key cancer driver genes [37, 38]. In this study, we describe the shortening of the MYC $3^{\prime} U T R$ in CRC, particularly in the more advanced disease (Fig. 1A). This finding suggests that many of the known MYC:miRNA interactions may not be present in certain cancer cells and highlights the need to expand the conventional focus on miRNA target sites in $3^{\prime}$ UTRs to include $5^{\prime}$ UTRs and coding regions, to map the full landscape of miRNA:target interactions in both normal and cancer cells. This will in turn facilitate the selection of the most promising miRNA: target interactions for the development of potential clinical applications in a disease-specific context. In addition, as $3^{\prime}$ UTRs are targeted by other regulators such as RNA-binding proteins (RBPs), their shortening may also lead to the loss of RBP-mediated regulation [39]. Future studies will be key to unravelling the impact of 3'UTR shortening and the concomitant loss of these RNA:RNA and RNA:protein interactions on the expression and function of key cancer genes [38].

The discovery of the heterogenous MYC transcript population in cancer cells led us to focus on the identification of miRNAs, which target the MYC CDS as this is present in all MYC transcript variants. We describe a previously uncharacterized role for the tumorsuppressive miR-138 as a potent regulator of MYC expression in both CRC and HCC (Fig. 6F). The combination of MYC $3^{\prime} U T R$ 
A

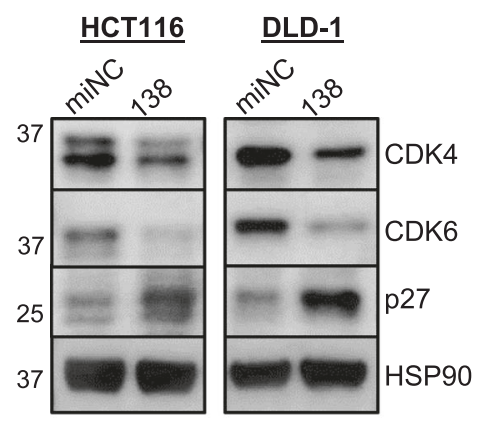

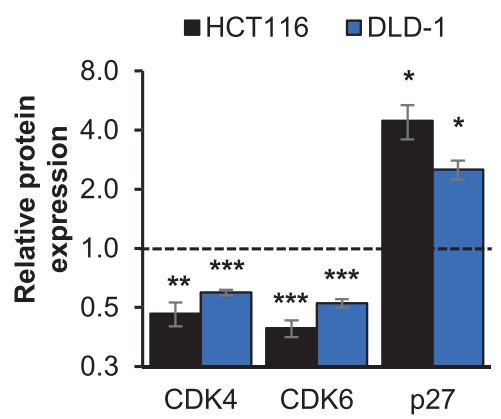

C
B

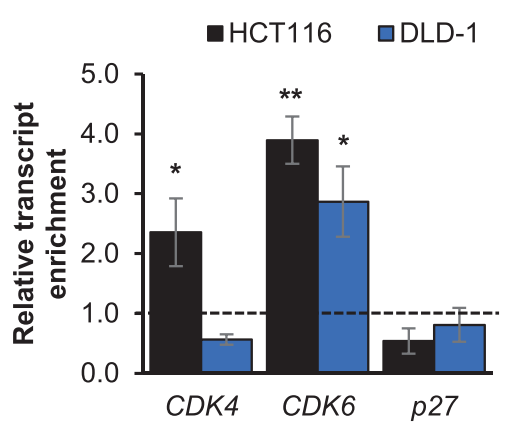

D
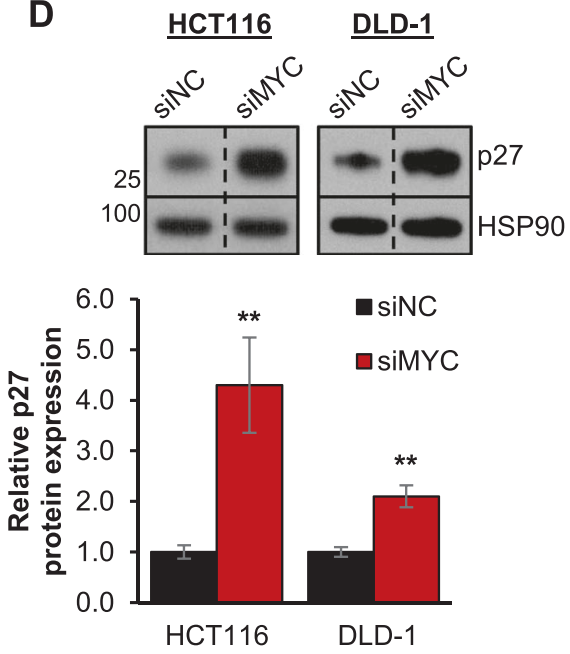

Fig. 3 MiR-138 regulates MYC target gene expression. A Western blot (left panel) and densitometry quantification (right panel) showing the protein expression of MYC target genes upon the overexpression of miR-138 (138) in HCT116 and DLD-1 cell lines. B RT-qPCR analysis of CDK4, CDK6 and p27 transcript enrichment upon biotinylated miR-138 pulldown in HCT116 and DLD-1 cell lines. C The sequences of the wild-type (WT) and mutant miR-138 MREs on the CDK6 transcript (top panel) and the effect of miR-138 overexpression on the luciferase activity of the MRE-reporter constructs (bottom panel) in HCT116 (left panel) and DLD-1 (right panel) cell lines. RC denotes the reverse complement of miR138 which was used as positive control. D Western blot (top panel) and densitometry quantification (bottom panel) of p27 protein expression upon the knockdown of MYC in HCT116 and DLD-1 cell lines. Mean \pm SEM; $N \geq 3$. ${ }^{*} P<0.05 ;{ }^{* *} P<0.01 ;{ }^{* * *} P<0.001$.

shortening and the downregulation of miR-138 may contribute to increased MYC expression in cancer cells. Low levels of miR-138 also directly or indirectly affect the expression of MYC target genes such as CDK6 and p27. Cumulatively, these effects promote uncontrolled cell proliferation and subsequent cancer development.

Critically, we show that treatment with miR-138 mimics was able to reduce MYC expression and tumor growth in a wellestablished transgenic mouse model for MYC-driven HCC. However, as miR-138 has been shown to exhibit context-specific tumor suppressive or oncogenic properties, it is important to examine its potential delivery into tissues in which it acts as an oncogenic miRNA. For example, miR-138 has been validated as a pro-survival oncogenic miRNA in malignant glioma [31]. In our experiments, we did not observe significant enrichment of miR138 in the brain upon mimic injection (Supplementary Fig. S4a). This is likely to be due to the blood-brain barrier, which diminishes the risk of malignant glioma development upon mimic administration. Interestingly, there was a moderate level of miR-138 enrichment in the kidney, suggesting that the mimics may be readily eliminated from the body hence minimizing potential chronic toxicity.
In recent years, there has been remarkable progress in the development of miRNAs as diagnostic and prognostic tools as well as therapeutic targets $[40,41]$. In addition, novel delivery systems such as viral vectors [42, 43], lipid-based nanocarriers [44, 45], cellderived membrane vesicles [46, 47], polymeric vectors [48-50], as well as 3D scaffold-based delivery systems [51] have significantly improved the delivery efficiency of both antisense miRNAs (antimiRs) and miRNA mimics to suppress or replenish miRNAs, respectively $[13,52]$. Some studies which have progressed into clinical trials for cancer treatment include that of miR-16 mimics (MesomiR1) (ClinicalTrials.gov identifier: NCT02369198) for the treatment of mesothelioma and NSCLC which is currently in Phase I clinical trials [53], and antimiR-155 (MRG-106) (ClinicalTrials.gov identifier: NCT02580552) for the treatment of T cell lymphoma and mycosis fungoides in Phase II clinical trials [54].

Despite these progressive breakthroughs in miRNA research, significant challenges remain before we can successfully translate them into clinical therapeutics. Firstly, as miRNAs may have different targets in different tissues and organs, it is critical to deliver the miRNA therapeutic specifically to the targeted tissue to avoid unintended side effects [55]. In addition, diverse epigenetic, transcriptional and hormonal mechanisms may underlie miRNA 
A

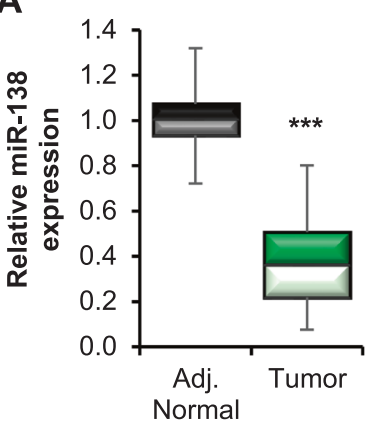

D

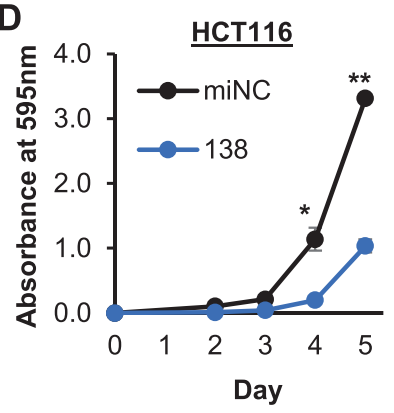

miNC

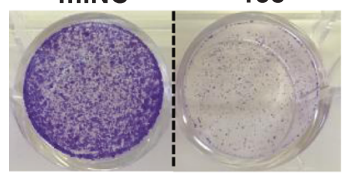

F
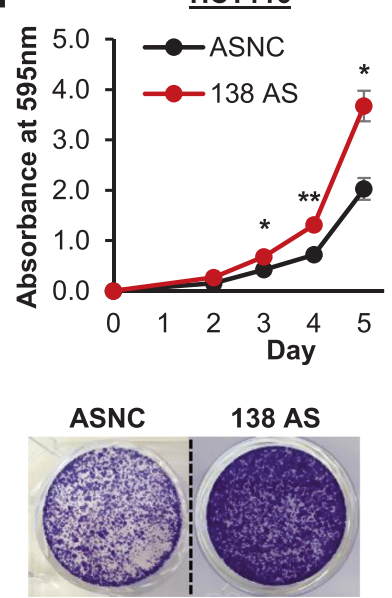

B

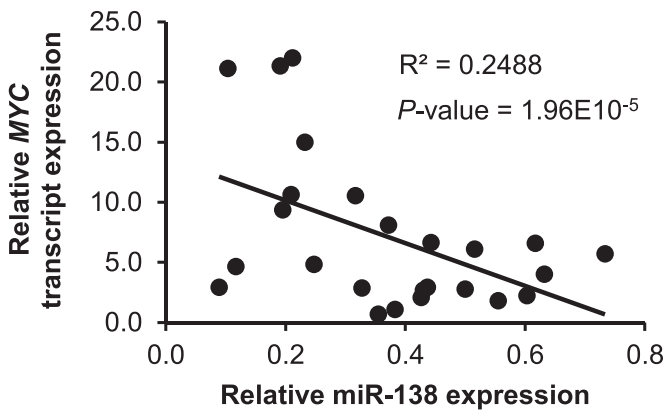

E

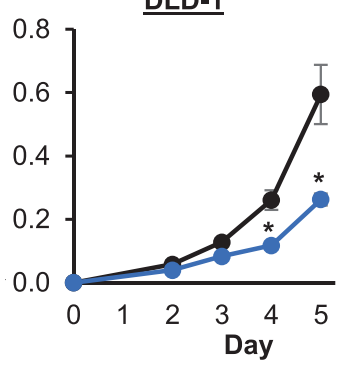

miNC

138

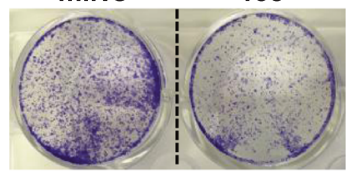

DLD-1

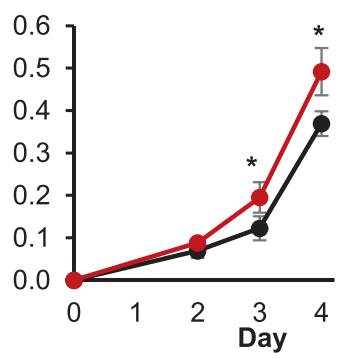

ASNC

138 AS

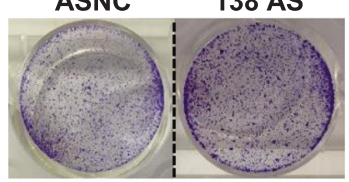

G

HCT116
C

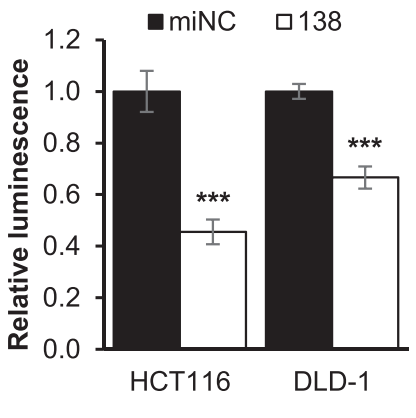

DLD-1

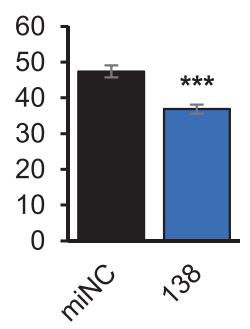

miNC 138

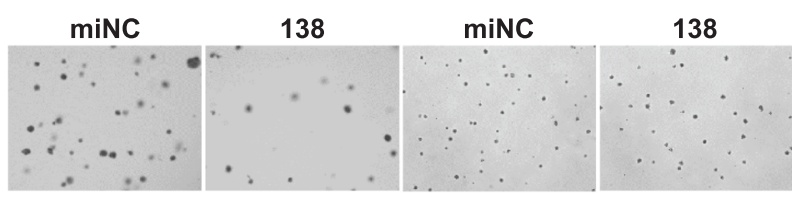

DLD-1
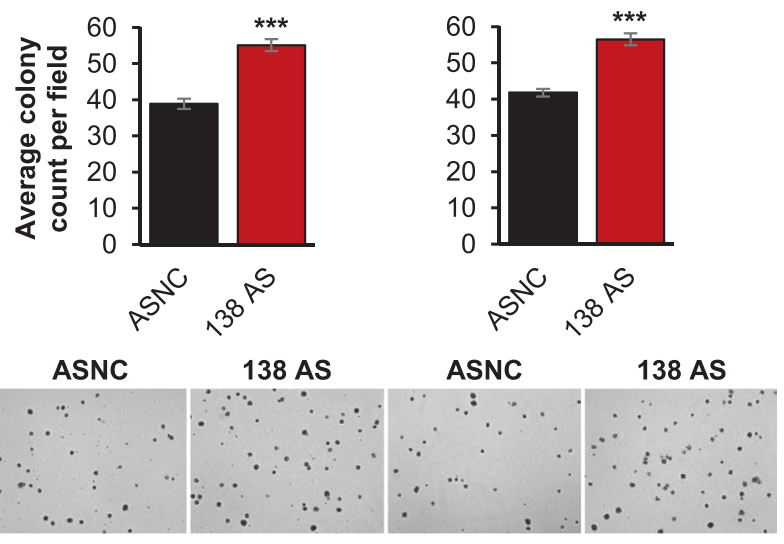

Fig. 4 MiR-138 possesses tumor-suppressive properties in CRC. A RT-qPCR quantification of miR-138 expression in tumor and adjacent normal samples from CRC patients $(N=25)$. B Correlation between miR-138 and MYC transcript expression in tumor samples of CRC patients $(N=25)$. C CellTiter-Glo assay examining the effect of miR-138 overexpression on the cell viability of HCT116 and DLD-1 cells. D, E Effect of miR-138 overexpression (138) on anchorage-dependent (D) and anchorage-independent (E) growth of HCT116 (left panel) and DLD-1 (right panel) cells. F, G Effect of miR-138 inhibition (138 AS) on anchorage-dependent (F) and anchorage-independent growth (G) of HCT116 (left panel) and DLD-1 (right panel) cells. The representative images of anchorage-dependent and -independent growth are shown at the bottom panels. C, E and G Mean \pm SEM; $N \geq 3$. D and F Mean \pm STD; $N \geq 3$. ${ }^{*} P<0.05 ;{ }^{* *} P<0.01 ;{ }^{* *} P<0.001$.

regulation, increasing the complexity of their therapeutic application [55]. Therefore, deeper insights into miRNA targeting patterns and efficient delivery in a tissue-specific manner will be key to unlocking the potential of miRNA therapeutics while minimizing off-target effects and the associated risks.

We anticipate that miRNA therapy will blossom into an integral part of the precision medicine toolbox in the future, and hope that our identification of the miR-138:MYC interaction may launch new avenues for the targeting of the heterogeneous MYC transcript population and the subsequent treatment of MYC-driven cancers.

\section{MATERIALS AND METHODS}

$3^{\prime}$ rapid amplification of CDNA ends ( $3^{\prime}$ RACE)

The $3^{\prime}$ RACE was performed using the RLM-RACE Kit (FirstChoice ${ }^{\circ}$ ) following the manufacturer's protocol. $1 \mu \mathrm{g}$ of total RNA from adjacent 
A

Liver Hepatocellular
Carcinoma

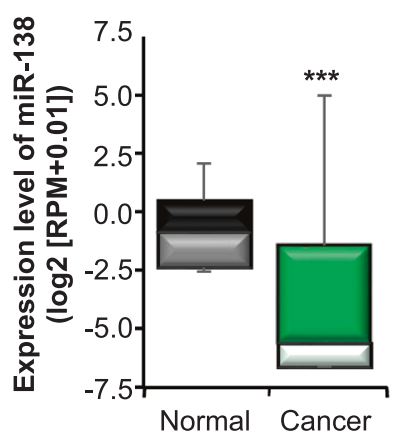

B

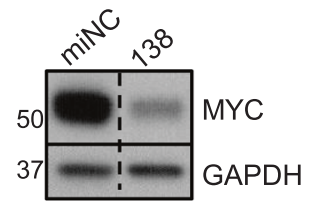

C
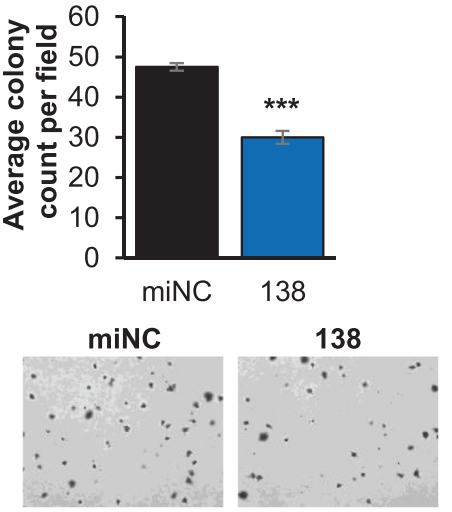

D

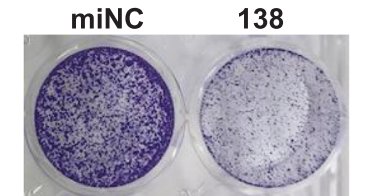

E

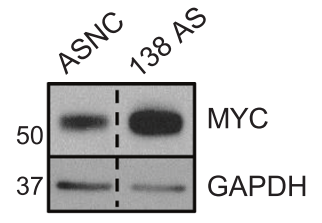

F

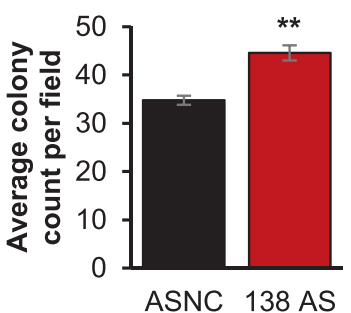

ASNC

\section{AS}

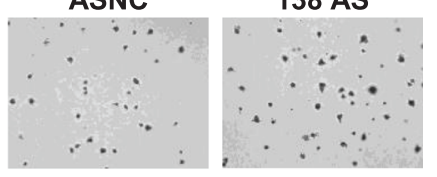

G

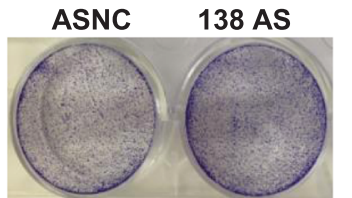

Fig. 5 MiR-138 downregulates MYC expression and inhibits growth in liver cancer. A MiR-138 expression in hepatocellular carcinoma (HCC) [50 normal versus 370 cancer] based on the TCGA dataset) [33]. B, C Effect of miR-138 overexpression (138) on MYC protein level (B) and the anchorage-independent growth (C) of HepG2 cells. Representative images of the anchorage-independent growth are shown at the bottom panel. D Representative image of anchorage-dependent growth of HepG2 cells upon miR-138 overexpression (138). E, F Effect of miR-138 inhibition (138 AS) on MYC protein level (E) and the anchorage-independent growth (F) of HepG2 cells. Representative images of the anchorage-independent growth are shown at the bottom panel. G Representative image of anchorage-dependent growth of HepG2 cells upon miR-138 inhibition (138 AS). Mean \pm SEM; $N \geq 3 .{ }^{* *} P<0.01 ;{ }^{* *} P<0.001$.

normal and tumor samples of CRC patients was used for the experiment. Primers used for the $3^{\prime}$ RACE are listed in Supplementary Table S4. The amplified complementary DNAs (cDNAs) were cloned into pcDNA4-TOPuromycin-mVenus-MAP. Sanger sequencing was performed on ten clones from each sample. The clones were analyzed for the length of the MYC $3^{\prime}$ UTR based on a few criteria; the presence of (1) both the forward and reverse inner PCR primers, (2) both the HindIII and BamHI restriction sites, and (3) the MYC CDS and stop codon preceding the $3^{\prime} U T R$. The poly $(A)$ at the end of the $3^{\prime} U T R$ was included in the calculation of the $3^{\prime} U T R$ length.

\section{Clinical samples}

CRC and adjacent normal tissues were obtained from the National University Hospital, Singapore. This study was approved by the relevant Institutional Review Boards in Singapore, and informed consent was obtained from all subjects. $10-20 \mathrm{mg}$ of fresh tissue was homogenized using a pestle plastic homogenizer and passed through a 21-gauge needle fitted to an RNase-free syringe. DNA, RNA, and protein were extracted using the QIAGEN AllPrep DNA/RNA/Protein Mini Kit according to the manufacturer's protocol.

\section{Reagents}

Reagents are as follows: anti-GAPDH (sc-47724), anti-HSP90 (sc-69703) and anti-YFP (sc-32897) antibodies (Santa Cruz Biotechnology); anti-MYC (ab32072) antibody (Abcam); anti-LYPLA1 (GTX104398) antibody (GeneTex); anti-p27 (3686S), anti-CDK4 (12790S) and anti-CDK6 (3136S) antibodies (Cell Signaling Technology (CST)); Rabbit IgG (301-001-003) antibody (Jackson ImmunoResearch Laboratories); miRIDIAN miRNA mimics for non-targeting control (NC) (miNC) and miRNAs (138, 139, 28, 99b, 193a, 197, 125a, 503, 744), miRIDIAN miRNA inhibitors for NC (ASNC), miR-28-5p (28 AS), miR-138 (138 AS), and miR-139-3p (139 AS), biotinylated miRNA reagents (NC and 138), siGENOME siRNA for NC (siNC) and MYC (siMYC), DharmaFECT 1 (Dharmacon), TRIZOL ${ }^{\circ}$, Lipofectamine 3000, Dulbecco's Modified Eagle Medium (DMEM), Roswell Park Memorial Institute (RPMI) 1640 medium, Opti-MEM ${ }^{\text {mm }}$ reduced serum media, fetal bovine serum (FBS), Dynabeads ${ }^{\mathrm{m} m} \mathrm{M}-280$ Streptavidin (ThermoFisher Scientific), psiCHECK-2 (Promega), pcDNA4-Puro (Addgene).

\section{Cell culture and transfection}

HCT116, HepG2, and Calu-1 cells were maintained in DMEM, while DLD-1, SNU-398 and 786-O cells were maintained in RPMI, supplemented with $10 \%$ FBS, $1 \%$ penicillin/streptomycin and 1\% L-glutamine. Both cell lines were grown at $37^{\circ} \mathrm{C}$ in a humidified atmosphere with $5 \% \mathrm{CO}_{2} . \mathrm{HCT} 116$ and DLD-1 cell lines were obtained from Horizon Discovery, 786-O, HepG2 and SNU-398 cell lines were obtained from ATCC, while Calu-1 cell line was a kind gift from Prof. Goh Boon Cher (Cancer Science Institute of Singapore). Mycoplasma contamination test was performed, and the results were negative. Cells were transfected with $50 \mathrm{nM}$ of miRNA mimics, inhibitors or siRNAs using DharmaFECT 1 as per the manufacturer's protocol at a seeding density of 150,000 cells per well in 12-well dishes. For miRNA inhibitors transfection, the cells were transfected in serum-free medium for $48 \mathrm{~h}$.

MS2-tagged RNA affinity purification (MS2- TRAP) pulldown HCT116 cells were seeded at a density of one million cells per well in sixwell plates. Cells were co-transfected with plasmids containing MS2binding protein (MS2-BP) fused with the yellow fluorescent protein (YFP) and the MYC CDS tagged with the MS2 hairpin sequence. The protocol was taken from the RIP-ChIP protocol [56].

\section{MicroRNA target prediction and databases}

MicroRNA target prediction was performed using RNA22 which is available at https://cm.jefferson.edu/rna22/Interactive/ [20]. Prediction of miR-138 MREs on the human MYC CDS was also performed using STarMir, which is available at https://sfold.wadsworth.org/cgi-bin/starmirtest2.pl and miRDB, which is available at http://mirdb.org/. MiR-138 targets were obtained from ENCORI (The Encyclopedia of RNA Interactomes), which is available at http://starbase.sysu.edu.cn/index.php [33]. The miR-138 targets were 
A

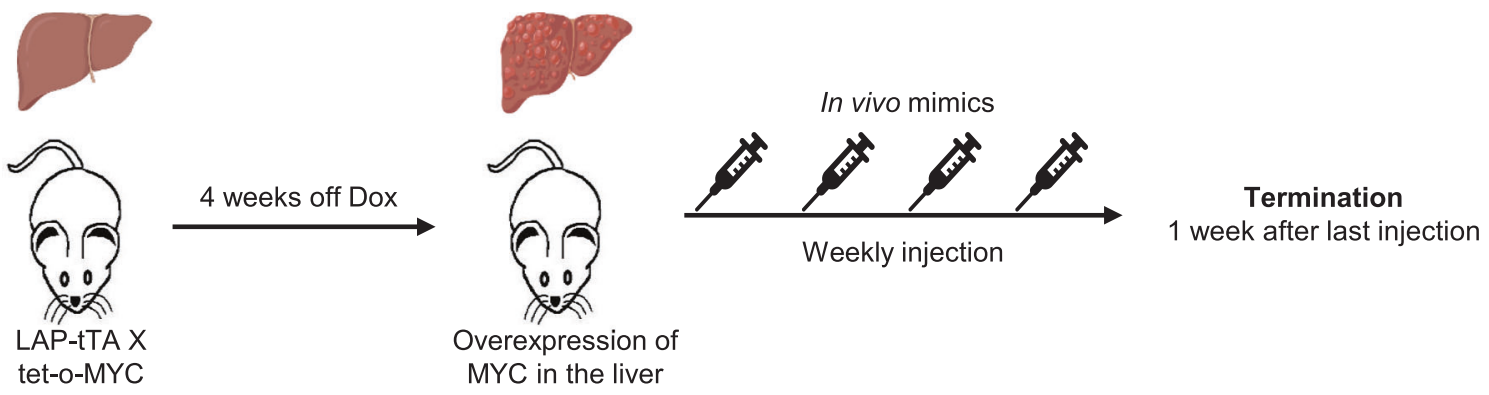

B

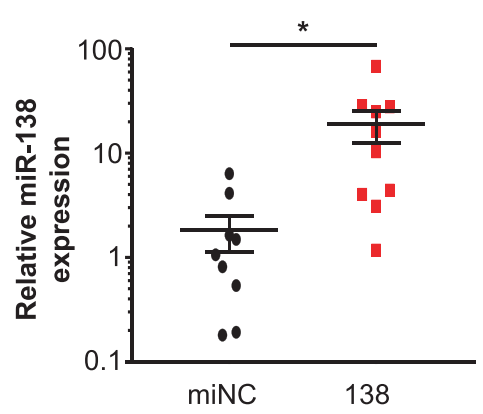

E
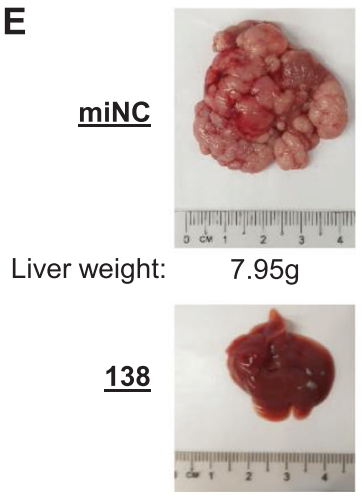

Liver weight:

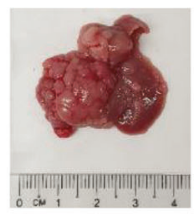

$3.30 \mathrm{~g}$

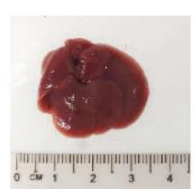

C

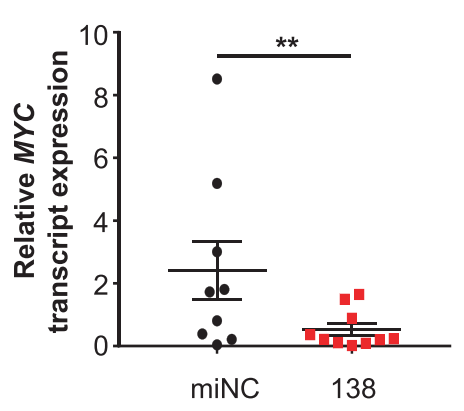

F

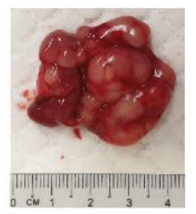

Found dead

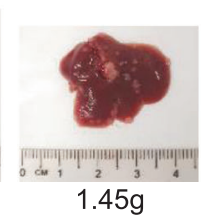

D

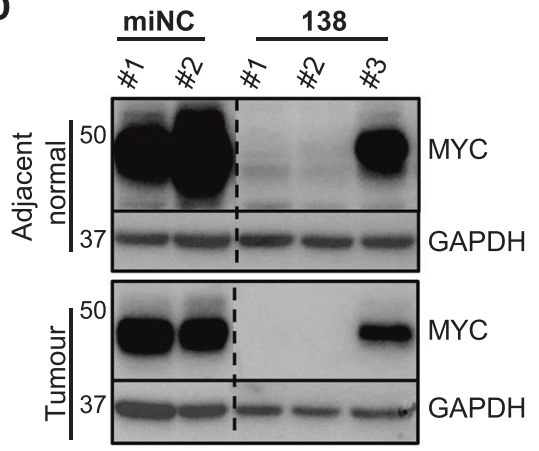

Fig. 6 MiR-138 suppresses MYC-driven carcinogenesis in vivo. A Schematic showing the timeline of the intravenous miR-138 mimic injection into transgenic mice. B, C RT-qPCR analyses of miR-138 (B) and MYC transcript (C) expression in the liver upon miNC and miR-138 mimic injection [9 miNC versus 10 miR-138 mimics injection]. D Representative Western blot analysis of MYC protein levels upon miNC and miR-138 mimic injection. E Representative images of the mouse livers upon miNC (top panel) and miR-138 (bottom panel) injection. (E - I) Samples were harvested one week after the final mimic injection. F Schematic diagram summarizing the effects of miR-138 on MYC and cancer development. Orange circle denotes miR-138 MRE. Mean \pm SEM. ${ }^{*} P<0.05 ;{ }^{* * P}<0.01$.

predicted by more than 4 prediction platforms and were downregulated in CRC and HCC.

\section{Protein extraction and western blot analysis}

Cells were trypsinized and harvested in the appropriate growth medium and lysed on ice for $10 \mathrm{~min}$ in protein lysis buffer (PLB) (10 mM HEPES pH 7.0, 0.1 M KCl, $5 \mathrm{mM} \mathrm{MgCl} 2,25 \mathrm{mM}$ EDTA pH 8.0, 0.5\% (v/v) NP-40, Proteinase Inhibitor, $20 \mu \mathrm{M}$ DTT), followed by centrifugation at $16,000 \times g$ for $15 \mathrm{~min}$. The concentrations of the lysates were measured using Bradford Protein Assay (Bio-Rad). 8-12 $\mu \mathrm{g}$ of protein lysates was separated on $4-12 \%$ Bis-Tris NuPAGE ${ }^{\circ}$ Precast gels (ThermoFisher Scientific) and transferred to PVDF membranes using the Mini Trans-Blot Electrophoretic Transfer Cell (BioRad) in transfer buffer (2.5 mM Tris, $19.2 \mathrm{mM}$ glycine and $10 \%(\mathrm{v} / \mathrm{v})$ methanol). The membranes were probed with specific primary antibodies followed by the corresponding secondary antibodies.
RNA extraction, real-time quantitative PCR (RT-qPCR)

Total RNA was isolated using the TRIZOL ${ }^{\circ}$ reagent and the PureLink ${ }^{\circ}$ RNA Mini Kit (Ambion) as per the manufacturer's protocol. $500 \mathrm{ng}$ of total RNA was used to synthesize the CDNA using the High-Capacity CDNA Reverse Transcription Kit (ThermoFisher Scientific). qPCR was performed using the PowerUp ${ }^{\text {TM }}$ SYBR $^{\circ}$ Green Master Mix (Applied Biosystems) on the QuantStudio 5 Real-Time PCR System. The primers used for RT-qPCR are listed in Supplementary Table S5.

\section{Plasmids}

Oligonucleotides for the MREs (Supplementary Table S6) were annealed by mixing $100 \mu \mathrm{M}$ of each primer in $5 \times$ sequencing and annealing buffer $(1 \mathrm{M}$ Tris $\mathrm{HCl} \mathrm{pH} \mathrm{7.5,5} \mathrm{M} \mathrm{NaCl}, 1 \mathrm{M} \mathrm{MgCl}_{2}$ ). The mixture was heated at $95^{\circ} \mathrm{C}$ for $5 \mathrm{~min}$ and cooled to room temperature and ligated into the digested psiCHECK-2 vector. 


\section{Luciferase reporter assays}

For dual-luciferase reporter assays, cells were seeded at a density of 50,000 cells per well in 24-well plates a day prior to transfection. $5 \mathrm{ng}$ of psiCHECK2 plasmids was co-transfected with $50 \mathrm{nM}$ miRNA mimics. At $48 \mathrm{~h}$ posttransfection, cells were washed in PBS, lysed and luminescence was measured following the manufacturer's instructions (Promega).

\section{Site-directed mutagenesis (SDM)}

SDM was performed using the QuikChange Lightning Multi Site-Directed Mutagenesis Kit (Agilent Technologies) following the manufacturer's protocol. SDM primers are listed in Supplementary Table S7.

\section{Biotinylated MiRNA pulldown}

HCT116 and DLD-1 cells were seeded at a density of 500,000 cells per well in six-well plates. Cells were transfected with biotinylated miRNA mimics (Dharmacon), for $48 \mathrm{~h}$ prior to harvesting in protein lysis buffer. The pulldown experiment was performed as previously described [57]. RNAs bound to the miRNA baits were harvested using Trizol LS and purified using phenol: chloroform: isoamyl (25:24:1), followed by chloroform: isoamyl (24:1) (Sigma-Aldrich).

\section{CellTiter-Glo luminescent cell viability}

Cells were transfected with $50 \mathrm{nM}$ of miRNA mimics and seeded at a density of 1000 cells per well in 96-well plates. After $48 \mathrm{~h}$ transfection, $50 \mu \mathrm{l}$ of CellTiter-Glo assay reagents (Promega) were added to each well and incubated for $5 \mathrm{~min}$ at room temperature before measuring the luminescence readings.

\section{Anchorage-dependent growth assay}

At $24 \mathrm{~h}$ of post-transfection, cells were split to five wells at 30,000 cells per well density in 12-well plates. Cells were fixed with $10 \%$ neutral buffered formalin solution (Sigma-Aldrich) from day 0 to day 5 (excluding day 1). On day 5 , cells were stained with $0.5 \%$ crystal violet (Sigma-Aldrich) for $5 \mathrm{~min}$. The excess stain was removed by rinsing with water. After $2 \mathrm{~h}$ of drying, the crystal violet stain was dissolved in $10 \%$ acetic acid (Sigma-Aldrich). The plates were incubated at room temperature for $1 \mathrm{~h}$ with shaking. The absorbance was measured at $595 \mathrm{~nm}$.

\section{Soft agar assay}

A $0.6 \%$ agarose base was prepared in six-well plates. $24 \mathrm{~h}$ after transfection, cells were trypsinized, resuspended and diluted to 15,000 cells per well in growth medium. The cells were mixed with agarose to obtain a $0.3 \%$ top layer agar which was added above the $0.6 \%$ base agar. The cells were maintained at $37^{\circ} \mathrm{C}$ and the growth medium was changed twice a week. Images were taken at $\times 4$ magnification once every 5 days up to 14 days and quantified using ImageJ.

\section{Transgenic mice}

Tet-o-MYC transgenic mice (JAX stock \#019376) [58] and LAP-tTA mice (JAX stock \#003563) [59] were purchased from The Jackson Laboratory. The two mouse strains were crossed to produce transgenic mice which carry both the tet-o-MYC and LAP-tTA genes [36]. To induce MYC transgene expression, the diet containing doxycycline was replaced with normal chow diet when mice were 4-5 weeks old. Male and female mice with both transgenes overexpressed human MYC CDS in the liver were used in this study. All animal works were approved by NUS Institutional Animal Care and Use Committee (IACUC), protocol number R19-0852.

\section{In vivo MiRNA mimics tail-vein injection}

mirVana ${ }^{\mathrm{m}}$ miRNA mimics, Negative Control \#1 (miNC) and mirVana miRNA mimic - hsa-miR-138-5p (138), were purchased from ThermoFisher Scientific. Invivofectamine ${ }^{\mathrm{TM}} 3.0$ reagent (ThermoFisher Scientific) was used for the delivery of $20 \mu \mathrm{g}$ of miRNA mimic per mouse, following the manufacturer's protocol $[60,61]$. Five mice were randomly allocated into each miNC or 138 group for each experimental set. The miRNA mimics were delivered intravenously into the mice every week with a total of four doses by the end of the experiment. One week after the final injection, the mice were sacrificed, and the organs were harvested for downstream biochemical analyses. Experiments, data collection and analysis were performed by three investigators, with at least one blinded investigator.

\section{Statistical analysis}

All in vitro experiments were performed three times independently. Sample size was chosen based on $\mathrm{N}=\frac{\log \beta}{\log p}$ whereby $\beta$ is 0.05 and $p$ is 0.5 , thus the sample size was 4.32 (round up to 5) [62]. Values calculated from multiple independent experiments were presented as mean \pm SEM, while data shown from a representative experiment were presented as mean \pm SD. Unpaired Student's $t$ test was used to analyze the statistical significance whereby $P$ values $<0.05$ were considered statistically significant. *, $P<0.05 ;{ }^{*}, P<0.01 ;{ }^{* *}, P<0.001$.

\section{REFERENCES}

1. Sandberg R, Neilson JR, Sarma A, Sharp PA, Burge CB. Proliferating cells express mRNAs with shortened 3 ' untranslated regions and fewer microRNA target sites. Science. 2008;320:1643-7.

2. Mayr C, Bartel DP. Widespread shortening of $3^{\prime} U T R$ s by alternative cleavage and polyadenylation activates oncogenes in cancer cells. Cell. 2009;138:673-84.

3. Misiewicz-Krzeminska I, Sarasquete ME, Vicente-Duenas C, Krzeminski P, Wiktorska K, Corchete LA, et al. Post-transcriptional Modifications Contribute to the Upregulation of Cyclin D2 in Multiple Myeloma. Clin Cancer Res. 2016;22:207-17.

4. Akman BH, Can T, Erson-Bensan AE. Estrogen-induced upregulation and 3'-UTR shortening of CDC6. Nucleic Acids Res. 2012;40:10679-88.

5. Nesbit CE, Tersak JM, Prochownik EV. MYC oncogenes and human neoplastic disease. Oncogene. 1999;18:3004-16.

6. Gabay M, Li Y, Felsher DW. MYC activation is a hallmark of cancer initiation and maintenance. Cold Spring Harb Perspect Med. 2014;4:a014241.

7. Rochlitz CF, Herrmann R, de Kant E. Overexpression and amplification of c-myc during progression of human colorectal cancer. Oncology. 1996;53:448-54.

8. Kozma L, Kiss I, Szakall S, Ember I. Investigation of c-myc oncogene amplification in colorectal cancer. Cancer Lett. 1994;81:165-9.

9. Fujiwara $Y$, Monden M, Mori T, Nakamura Y, Emi M. Frequent multiplication of the long arm of chromosome 8 in hepatocellular carcinoma. Cancer Res. 1993;53:857-60.

10. Ahn SM, Jang SJ, Shim JH, Kim D, Hong SM, Sung CO, et al. Genomic portrait of resectable hepatocellular carcinomas: implications of RB1 and FGF19 aberrations for patient stratification. Hepatology. 2014;60:1972-82.

11. Smith DR, Myint T, Goh HS. Over-expression of the c-myc proto-oncogene in colorectal carcinoma. Br J Cancer. 1993;68:407-13.

12. Mihailovich $M$, Bremang $M$, Spadotto V, Musiani D, Vitale $E$, Varano $G$, et al. miR17-92 fine-tunes MYC expression and function to ensure optimal B cell lymphoma growth. Nat Commun. 2015;6:8725.

13. Rupaimoole R, Slack FJ. MicroRNA therapeutics: towards a new era for the management of cancer and other diseases. Nat Rev Drug Disco. 2017;16:203-22.

14. Jackstadt R, Hermeking $H$. MicroRNAs as regulators and mediators of c-MYC function. Biochim Biophys Acta. 2015;1849:544-53.

15. Shams R, Asadzadeh Aghdaei H, Behmanesh A, Sadeghi A, Zali M, Salari S, et al. MicroRNAs Targeting MYC Expression: Trace of Hope for Pancreatic Cancer Therapy. A Systematic Review. Cancer Manag Res. 2020;12:2393-404.

16. Wang YB, Zhao XH, Li G, Zheng JH, Qiu W. MicroRNA-184 inhibits proliferation and promotes apoptosis of human colon cancer SW480 and HCT116 cells by downregulating C-MYC and BCL-2. J Cell Biochem. 2018;119:1702-15.

17. Wang H, Cao F, Li X, Miao H, E J, Xing J, et al. miR-320b suppresses cell proliferation by targeting c-Myc in human colorectal cancer cells. BMC Cancer. 2015;15:748.

18. Xie F, Yuan $Y$, Xie L, Ran $P$, Xiang $X$, Huang $Q$, et al. miRNA-320a inhibits tumor proliferation and invasion by targeting c-Myc in human hepatocellular carcinoma. Onco Targets Ther. 2017;10:885-94.

19. Lin F, Ding R, Zheng S, Xing D, Hong W, Zhou Z, et al. Decrease expression of microRNA-744 promotes cell proliferation by targeting c-Myc in human hepatocellular carcinoma. Cancer Cell Int. 2014;14:58.

20. Miranda KC, Huynh T, Tay Y, Ang YS, Tam WL, Thomson AM, et al. A pattern-based method for the identification of MicroRNA binding sites and their corresponding heteroduplexes. Cell. 2006;126:1203-17.

21. Hermeking $H$, Rago C, Schuhmacher M, Li Q, Barrett JF, Obaya AJ, et al. Identification of CDK4 as a target of c-MYC. Proc Natl Acad Sci USA. 2000;97:2229-34.

22. Mateyak MK, Obaya AJ, Sedivy JM. c-Myc regulates cyclin D-Cdk4 and -Cdk6 activity but affects cell cycle progression at multiple independent points. Mol Cell Biol. 1999;19:4672-83.

23. Yang W, Shen J, Wu M, Arsura M, FitzGerald M, Suldan Z, et al. Repression of transcription of the p27(Kip1) cyclin-dependent kinase inhibitor gene by c-Myc. Oncogene. 2001;20:1688-702.

24. Li D, Song H, Wu T, Xie D, Hu J, Zhao J, et al. MiR-138-5p targeting LIMK1 suppresses breast cancer cell proliferation and motility. RSC Adv. 2017;7:52030-8. 
25. Qiu S, Huang D, Yin D, Li F, Li X, Kung HF, et al. Suppression of tumorigenicity by microRNA-138 through inhibition of EZH2-CDK4/6-pRb-E2F1 signal loop in glioblastoma multiforme. Biochim Biophys Acta. 2013;1832:1697-707.

26. Mohammed A, Zhang C, Zhang S, Shen Q, Li J, Tang Z, et al. Inhibition of cell proliferation and migration in nonsmall cell lung cancer cells through the suppression of LYPLA1. Oncol Rep. 2019;41:973-80.

27. You C, Jin L, Xu Q, Shen B, Jiao X, Huang X. Expression of miR-21 and miR-138 in colon cancer and its effect on cell proliferation and prognosis. Oncol Lett. 2019;17:2271-7.

28. Long L, Huang G, Zhu H, Guo Y, Liu Y, Huo J. Down-regulation of miR-138 promotes colorectal cancer metastasis via directly targeting TWIST2. J Transl Med. 2013;11:275.

29. Xu Y, Pan ZG, Shu L, Li QJ. Podocalyxin-like, targeted by miR-138, promotes colorectal cancer cell proliferation, migration, invasion and EMT. Eur Rev Med Pharm Sci. 2018;22:8664-74.

30. Zhao L, Yu H, Yi S, Peng X, Su P, Xiao Z, et al. The tumor suppressor miR-138-5p targets PD-L1 in colorectal cancer. Oncotarget. 2016;7:45370-84.

31. Chan XH, Nama S, Gopal F, Rizk P, Ramasamy S, Sundaram G, et al. Targeting glioma stem cells by functional inhibition of a prosurvival oncomiR-138 in malignant gliomas. Cell Rep. 2012;2:591-602.

32. Nama S, Muhuri M, Di Pascale F, Quah S, Aswad L, Fullwood M, et al. MicroRNA138 is a Prognostic Biomarker for Triple-Negative Breast Cancer and Promotes Tumorigenesis via TUSC2 repression. Sci Rep. 2019;9:12718.

33. Li JH, Liu S, Zhou H, Qu LH, Yang JH. starBase v2.0: decoding miRNA-ceRNA, miRNA-ncRNA and protein-RNA interaction networks from large-scale CLIP-Seq data. Nucleic Acids Res. 2014;42:D92-97.

34. Chan KL, Guan XY, Ng IO. High-throughput tissue microarray analysis of c-myc activation in chronic liver diseases and hepatocellular carcinoma. Hum Pathol. 2004;35:1324-31.

35. Wu KJ, Grandori C, Amacker M, Simon-Vermot N, Polack A, Lingner J, et al. Direct activation of TERT transcription by c-MYC. Nat Genet. 1999;21:220-4.

36. Shachaf CM, Kopelman AM, Arvanitis C, Karlsson A, Beer S, Mandl S, et al. MYC inactivation uncovers pluripotent differentiation and tumour dormancy in hepatocellular cancer. Nature. 2004;431:1112-7.

37. Park HJ, Ji P, Kim S, Xia Z, Rodriguez B, Li L, et al. 3'UTR shortening represses tumorsuppressor genes in trans by disrupting ceRNA crosstalk. Nat Genet. 2018;50:783-9.

38. Desi N, Tay Y. The Butterfly Effect of RNA Alterations on Transcriptomic Equilibrium. Cells. 2019;8:1634-1656.

39. Berkovits BD, Mayr C. Alternative 3'UTRs act as scaffolds to regulate membrane protein localization. Nature. 2015;522:363-7.

40. Beg MS, Brenner AJ, Sachdev J, Borad M, Kang YK, Stoudemire J, et al. Phase I study of MRX34, a liposomal miR-34a mimic, administered twice weekly in patients with advanced solid tumors. Invest N Drugs. 2017;35:180-8.

41. Xu X, Chen W, Miao R, Zhou Y, Wang Z, Zhang L, et al. miR-34a induces cellular senescence via modulation of telomerase activity in human hepatocellular carcinoma by targeting FoxM1/c-Myc pathway. Oncotarget. 2015;6:3988-4004.

42. Miyazaki $Y$, Adachi $H$, Katsuno $M$, Minamiyama $M$, Jiang $Y M$, Huang $Z$, et al. Viral delivery of miR-196a ameliorates the SBMA phenotype via the silencing of CELF2. Nat Med. 2012;18:1136-41.

43. Ramanujam D, Sassi Y, Laggerbauer B, Engelhardt S. Viral Vector-Based Targeting of miR-21 in Cardiac Nonmyocyte Cells Reduces Pathologic Remodeling of the Heart. Mol Ther. 2016;24:1939-48.

44. Zhang Q, Ran R, Zhang L, Liu Y, Mei L, Zhang Z, et al. Simultaneous delivery of therapeutic antagomirs with paclitaxel for the management of metastatic tumors by a pH-responsive anti-microbial peptide-mediated liposomal delivery system. J Control Release. 2015;197:208-18.

45. Endo-Takahashi Y, Negishi Y, Nakamura A, Ukai S, Ooaku K, Oda Y, et al. Systemic delivery of miR-126 by miRNA-loaded Bubble liposomes for the treatment of hindlimb ischemia. Sci Rep. 2014;4:3883.

46. Wang Y, Chen X, Tian B, Liu J, Yang L, Zeng L, et al. Nucleolin-targeted Extracellular Vesicles as a Versatile Platform for Biologics Delivery to Breast Cancer. Theranostics. 2017;7:1360-72.

47. Cui J, Li Q, Luo M, Zhong Z, Zhou S, Jiang L, et al. Leukemia cell-derived microvesicles induce $\mathrm{T}$ cell exhaustion via miRNA delivery. Oncoimmunology. 2018;7:e1448330.

48. Huang Z, Shi T, Zhou Q, Shi S, Zhao R, Shi H, et al. miR-141 Regulates colonic leukocytic trafficking by targeting CXCL12beta during murine colitis and human Crohn's disease. Gut. 2014;63:1247-57.

49. Gao S, Tian H, Guo Y, Li Y, Guo Z, Zhu X, et al. miRNA oligonucleotide and sponge for miRNA-21 inhibition mediated by PEI-PLL in breast cancer therapy. Acta Biomater. 2015;25:184-93.

50. Biray Avci C, Ozcan I, Balci T, Ozer O, Gunduz C. Design of polyethylene glycolpolyethylenimine nanocomplexes as non-viral carriers: mir-150 delivery to chronic myeloid leukemia cells. Cell Biol Int. 2013;37:1205-14.
51. Conde J, Oliva N, Atilano M, Song HS, Artzi N. Self-assembled RNA-triple-helix hydrogel scaffold for microRNA modulation in the tumour microenvironment. Nat Mater. 2016;15:353-63.

52. Fu Y, Chen J, Huang Z. Recent progress in microRNA-based delivery systems for the treatment of human disease. ExRNA. 2019;1:24-38.

53. Reid G, Pel ME, Kirschner MB, Cheng YY, Mugridge N, Weiss J, et al. Restoring expression of miR-16: a novel approach to therapy for malignant pleural mesothelioma. Ann Oncol. 2013;24:3128-35.

54. Seto AG, Beatty X, Lynch JM, Hermreck M, Tetzlaff M, Duvic M, et al. Cobomarsen, an oligonucleotide inhibitor of miR-155, co-ordinately regulates multiple survival pathways to reduce cellular proliferation and survival in cutaneous T-cell lymphoma. Br J Haematol. 2018;183:428-44.

55. Zhang S, Cheng Z, Wang Y, Han T. The Risks of miRNA Therapeutics: In a Drug Target Perspective. Drug Des Devel Ther. 2021;15:721-33.

56. Yoon JH, Srikantan S, Gorospe M. MS2-TRAP (MS2-tagged RNA affinity purification): tagging RNA to identify associated miRNAs. Methods. 2012;58:81-7.

57. Lal A, Thomas MP, Altschuler G, Navarro F, O'Day E, Li XL, et al. Capture of microRNA-bound mRNAs identifies the tumor suppressor miR-34a as a regulator of growth factor signaling. PLoS Genet. 2011;7:e1002363.

58. Felsher DW, Bishop JM. Reversible tumorigenesis by MYC in hematopoietic lineages. Mol Cell. 1999;4:199-207.

59. Kistner A, Gossen M, Zimmermann F, Jerecic J, Ullmer C, Lubbert $H$, et al. Doxycycline-mediated quantitative and tissue-specific control of gene expression in transgenic mice. Proc Natl Acad Sci USA. 1996;93:10933-8.

60. Chu-Tan JA, Fernando N, Aggio-Bruce R, Cioanca AV, Valter K, Andronikou N, et al. A method for gene knockdown in the retina using a lipid-based carrier. Mol Vis. 2020;26:48-63.

61. Migliore C, Martin V, Leoni VP, Restivo A, Atzori L, Petrelli A, et al. MiR-1 downregulation cooperates with MACC1 in promoting MET overexpression in human colon cancer. Clin Cancer Res. 2012;18:737-47.

62. Dell RB, Holleran S, Ramakrishnan R. Sample size determination. ILAR J. 2002;43:207-13.

\section{ACKNOWLEDGEMENTS}

We thank all past and present YT lab members for their constructive feedback on this project. We also thank MiRXES for the high-throughput qPCR analysis. We apologize to all colleagues whose work could not be cited due to space constraints. YT is funded by a Singapore National Research Foundation Fellowship, a National University of Singapore President's Assistant Professorship, a NMRC OF-IRG, and a Joint NCIS and NUS Cancer Programme Seed Grant. ND is supported by the NUS Research Scholarship. This research is supported by the National Research Foundation Singapore and the Singapore Ministry of Education under its Research Centres of Excellence initiative, as well as the RNA Biology Center at the Cancer Science Institute of Singapore, NUS, as part of funding under the Singapore Ministry of Education's AcRF Tier 3 grants, Grant number MOE2014-T3-1-006.

\section{AUTHOR CONTRIBUTIONS}

YT conceptualized and supervised the study. ND and VT designed and performed experiments and analyzed data. QYT, CYL, HT and XHC assisted in the collection of mouse samples and the processing of CRC patient samples. ASM performed the MS2TRAP experiment. QYT, HT, JJC, BZ and PP performed experiments. BES, SW, KCL, CSC, WKC, BL, IJWT and KKT provided the CRC patient samples. ND, VT, JJC and YT prepared the paper.

\section{COMPETING INTERESTS}

The authors declare no competing interests.

\section{ADDITIONAL INFORMATION}

Supplementary information The online version contains supplementary material available at https://doi.org/10.1038/s41388-021-02084-x.

Correspondence and requests for materials should be addressed to Yvonne Tay.

Reprints and permission information is available at http://www.nature.com/ reprints

Publisher's note Springer Nature remains neutral with regard to jurisdictional claims in published maps and institutional affiliations. 
Open Access This article is licensed under a Creative Commons At c) Atribution 4.0 International License, which permits use, sharing,
adaptation, distribution and reproduction in any medium or format, as long as you give appropriate credit to the original author(s) and the source, provide a link to the Creative Commons license, and indicate if changes were made. The images or other third party material in this article are included in the article's Creative Commons license, unless indicated otherwise in a credit line to the material. If material is not included in the article's Creative Commons license and your intended use is not permitted by statutory regulation or exceeds the permitted use, you will need to obtain permission directly from the copyright holder. To view a copy of this license, visit http://creativecommons. org/licenses/by/4.0/.

(c) The Author(s) 2021 